\title{
New AFC Algorithm For A Fully-Digital MDPSK DS/CDMA Receiver
}

\author{
Jing Lei and Tung-Sang $\mathrm{Ng}$ \\ Department of Electrical and Electronic Engineering, The University of Hong Kong \\ Pokfulam Road, Hong Kong \\ Phone: +852-28578418, Fax: +852-25598738, email: jlei@eee.hku.hk
}

\begin{abstract}
A new AFC algorithm is presented in this paper for correcting the sizable carrier frequency offset of a fully-digital MDPSK DS/CDMA receiver, which explores the power degradation at the correlator output and has an acquisition range of twice the symbol rate. To achieve fast frequency acquisition, the momentum steepest descent method and modified S-curve are employed in the algorithm. The simplicity of the algorithm makes it desirable for VLSI implementation. The performance of the proposed algorithm is also evaluated, and is compared with the Cramer-Rao bound and the results of other algorithms available in the literature.
\end{abstract}

\section{INTRODUCTION}

In direct-sequence code division multiple access (DS/CDMA) systems, M-ary differential phase shift keying (MDPSK) is widely used because of its high modulation efficiency and its elimination of the need for coherent phase recovery [1]. To correct the frequency offset arising from transceiver oscillator instability and Doppler shift, automatic frequency control (AFC) is required by the MDPSK DS/CDMA receiver. As the PN code synchronization of a DS/CDMA system can be accomplished with a rough estimation of the carrier frequency [1]-[2], AFC techniques applied in narrow-band communications can be employed for carrier frequency recovery after de-spreading [3]-[8]. However, their performances may not meet the requirements of future MDPSK DS/CDMA receivers such as wide acquisition range, fast acquisition, fully digital realization and low implementation complexity. For example, the acquisition ranges of rotational AFC [3], discrete-Fourier-transform AFC [3] and maximum-likelihood AFC [4] are $1 /(M T)$, where $M$ and $T$ denote the modulation order and the symbol spacing, respectively. This range is insufficient when $M$ is large and the frequency offset is on the order of the symbol rate, such as in low-earth-orbit satellite communications. Besides, the decision dependent AFC methods of [5] lose their usefulness when correct decisions cannot be obtained due to the initial frequency offset that is often sizable. Although dual-filter AFC [6] and balanced-quadricorrelator AFC [6] have acquisition ranges on the order of the symbol rate, both approaches involve complicated filter design to suppress the self noise and require precise knowledge of the channel. Two recent approaches are presented in [7] and [8]. Since either on-line matrix inversion or two-step Kalman filtering is necessary in their realization, their performance improvements are gained at the expense of high implementation complexity. In [2], Mauss et al. have generalized different AFC schemes previously used for fully-digital DS/CDMA receivers, such as decision-aided feedforward scheme (DAFF), non-decision-aided feedforward scheme (NDAFF) and nondecision-aided feedback scheme (NDAFB). These schemes employ phase-based algorithms, invoking analysis of the phase rotation to estimate the frequency offset and requiring a large look-up table for realization. Subject to the modulo- $2 \pi$ phase ambiguity, their frequency acquisition ranges are less than the symbol rate.

In this paper, we present a non-phase-based AFC algorithm with a wide acquisition range that is twice the symbol rate. By investigating the power degradation at the output of the PN code correlator, the problem of frequency acquisition is simplified into the recursive maximization of a cost function of the frequency offset. Momentum steepest descent method (MSDM) and modified S-curve are employed in the algorithm to speed up acquisition. The rest of this paper is organized as follows. Section 2 describes the receiver model. Section 3 discusses the derivation and the implementation of the proposed algorithm. The performance is also evaluated, and is compared with the Cramer-Rao bound (CRB) as well as the schemes given by [2]. Section 4 concludes the paper.

\section{RECEIVER MODEL}

Suppose the MDPSK signal is spreaded by PN code sequence $\left\{c_{1}, c_{2}, \ldots c_{N-1}\right\}$ and transmitted over a unity-gain AWGN channel with delay $\tau$ and phase shift $\theta$. Assume the chip pulse $g(t)$ satisfies the Nyquist pulse-shaping criterion for zero ISI [9]. Sampling the output of the receiver filter at chip rate $1 / T_{c}$ yields

$$
r_{r}=e^{j\left[2 \pi f_{d}\left(1 T_{c}+\hat{\tau}-\tau\right)+\theta\right]} \sum_{i=-\infty}^{\infty} e^{j \omega_{i}} \sum_{i=0}^{N-1} c_{n} g\left(v T_{c}-n T_{c}-l T+\hat{\tau}-\tau\right)+\tilde{w}_{r}
$$

where $f_{d}$ is the frequency offset between the carrier and the local oscillator, $\phi_{1}$ is differentially-encoded data, and $\tilde{w}_{v}$ is the sample of the complex Gaussian noise, which can be represented by $\tilde{w}_{I, v}+j \tilde{w}_{Q, v}$. It follows that $\tilde{w}_{l, r}$ and $\tilde{w}_{Q, r}$ are mutually independent real Gaussian noise having zero mean and the same variance, say $\sigma_{0}^{2}$. The sequence $\left\{r_{v}\right\}$ is processed by the PN code correlator and the $k$ th correlator output is given by

$$
Z_{k}=\frac{1}{N} \sum_{v=k N}^{(k+1) N-1} r_{v} c_{v-k N}
$$

In the absence of timing error, i.e. $\hat{\tau}=\tau,(2)$ reduces to

$$
Z_{k}=\alpha \exp \left[j\left(\tilde{\theta}_{k}+2 \pi f_{d} k T\right)\right]+w_{k} .
$$

where $\tilde{\theta}_{k}=\theta+\pi f_{d}\left(T-T_{c}\right)+\phi_{k} \quad, \quad w_{k}=\frac{1}{N} \sum_{v=k N N}^{(k+1, N-1} \tilde{w}_{,} c_{1-k i k}$ and 


$$
\alpha=\frac{\sin \left(\pi f_{d} T\right)}{N \sin \left(\pi f_{d} T_{c}\right)}
$$

\section{AFC ALGORITHM}

\subsection{Derivation}

As seen from (3), the power of $Z_{k}$ is proportional to $\alpha^{2}$ and we hence define $\alpha^{2}$ as the factor of power degradation. Fig.l shows the relationship between $\alpha^{2}$ and $f_{d} T$, the normalized frequency offset, with $N$ as the parameter. For $f_{d} T \in(-1,1)$, it is noted that $\alpha^{2}$ is almost independent of $N$ and decreases monotonically as $\left|f_{d} T\right|$ increases. Since the objective of AFC is to bring $f_{d}$ towards zero, maximization of $\alpha^{2}$ with respect to $f_{d}$ will eventually lead to $\alpha^{2}=1$ and $f_{d}=0$, the desired result, provided the initial frequency offset is within $(-1 / \mathrm{T}, 1 / \mathrm{T})$.

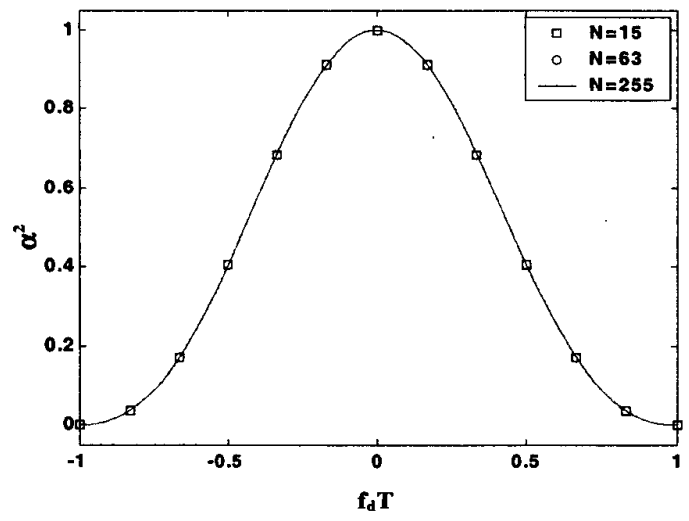

Figure 1. Relationship between power degradation factor and normalized frequency offset

Considering this observation, we adopt $\psi=\left|Z_{k}\right|^{2}$ as the cost function of $f_{d}$ and simplify the problem of frequency acquisition into the recursive maximization of $\psi$. Various methods including MSDM [10] can be applied in the iteration formula. Since MSDM converges much faster than ordinary gradient methods, it can speed up the acquisition. We employ it in the iteration formula and obtain the following equation:

$$
f_{d, k+1}=(\rho+1) f_{d, k}-\rho f_{d, k-1}+\mu \nabla_{k}, \quad|\rho|<1
$$

where $\left.f_{d, k} \stackrel{\Delta}{=} f_{d}\right|_{t=k T}, \mu$ is the step size and $\nabla_{k}$ is the gradient defined by $\nabla_{k}=\frac{-1}{4 \pi T_{c}} \cdot \frac{\partial \psi}{\partial f_{d . k}}$. Substituting (2) into $\psi$ and letting: $\quad \bar{r}_{k}=\left[\begin{array}{lllll}r_{k N} & r_{k N+1} & r_{k N+2} & \cdots & r_{1 k+1 N-1}\end{array}\right] \quad, \quad\left\{\bar{c}_{n}=n c_{n} \mid 0 \leq n \leq N-1\right\}$ and $\vec{C}_{w}=\left[\begin{array}{lllll}\bar{c}_{0} & \bar{c}_{1} & \bar{c}_{2} & \cdots & \bar{c}_{N-1}\end{array}\right]^{T}$, we have

$$
\nabla_{k}=\operatorname{Im}\left(Z_{k} W_{k}^{*}\right)
$$

where $\operatorname{Im}($.$) is the imaginary part, * is the conjugate operator and$

$$
W_{k}=\frac{1}{N}\left(\vec{r}_{k} \bullet \vec{C}_{w}\right)
$$

with • denoting the dot product of vectors.

The S-curve determines the acquisition performance of AFC and can be expressed as a function of $f_{d}$, say $S\left(f_{d}\right)$ [5]. In the absence of timing error, the S-curve for $(5)$ is

$$
S\left(f_{d}\right) \stackrel{\overrightarrow{=}}{=} E\left[\nabla_{f_{d}}\right]=\frac{-1}{N^{2}} \operatorname{Im}\left[\frac{1-e^{j 2 \pi f_{d} T}}{1-e^{j 2 \pi f_{d} T_{c}}} \sum_{m_{1}=0}^{N-1} m_{1} e^{-j 2 \pi m_{2} f_{d} T_{c}}\right] .
$$

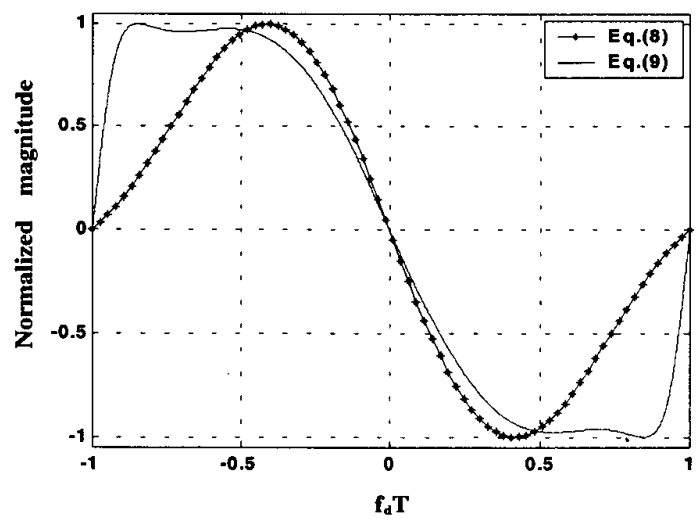

Figure 2. S-curve given by Eq.(8) and Eq.(9)

The S-curve given by (8) is normalized and depicted in Fig.2 for $f_{d} T \in(-1,1)$. However, a noticeable disadvantage accompanying this curve is that its magnitude decreases when $\left|f_{d}\right|$ goes beyond a certain threshold but is still far from $1 / T$. As a result, even if $\left|f_{d}\right|$ larger than this threshold can be tracked and brought to the vicinity of zero by (5), this process is lengthy due to the small-scale correction force of $S\left(f_{d}\right)$ at the beginning. This shortcoming can be circumvented by making some modifications to the shape of $S\left(f_{d}\right)$, such as pushing the threshold more closely to $1 / T$ and making the magnitude of the modified S-curve more uniformly distributed over the range being considered. For this purpose, we multiply ( 8 ) with a function $\chi\left(f_{d}\right)$ and the modified S-curve can be given by

$$
\bar{S}\left(f_{d}\right)=\chi\left(f_{d}\right) \cdot S\left(f_{d}\right)
$$

The choice of $\chi\left(f_{d}\right)$ is not unique. A simple approach is to let

$$
\chi\left(f_{d, k}\right)=\beta_{0}+\frac{\beta_{1}}{\left|Z_{k}^{2}\right|+\gamma}
$$


where $\left\{\beta_{0}, \beta_{1}, \gamma\right\}$ are positive constants. For instance, we choose $\left\{\beta_{0}=0.7, \beta_{l}=2, \gamma=0.2\right\}$ and plot (9) against (8) in Fig.2. Compared with $S\left(f_{d}\right), \bar{S}\left(f_{d}\right)$ has a much larger threshold and more uniform shape, which contributes to its superior performance to be demonstrated in section 3.3.

Inserting (10) into (5) finally yields the proposed AFC algorithm:

$$
f_{d . k+1}=(\rho+1) f_{d . k}-\rho f_{d . k-1}+\mu \chi\left(f_{d . k}\right) \nabla_{k}
$$

\subsection{Implementation}

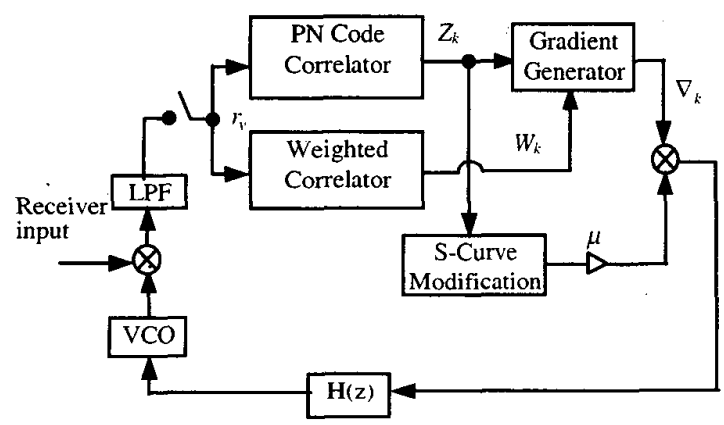

Figure 3. Block diagram of the proposed AFC Algorithm

Fig.3 depicts the block diagram for the proposed algorithm. The PN code correlator and the weighted correlator generate gradient $\nabla_{k}$ in line with (2), (6) and (7). The only difference between the weighted correlator and the PN code correlator is that the former has the weighted code sequence of $\left\{n c_{n}\right\}$. As observed from (7), $W_{k}$ can be obtained similarly to $Z_{k}$ by passing $r_{\text {, through the }}$ weighted correlator. Besides, as expressed in (10), the S-curve modification factor $\chi\left(f_{d}\right)$ is produced by exploiting the PN code correlator output $Z_{k}$. Then, the gradient component is multiplied by the factor $\chi\left(f_{d}\right)$ and the step size $\mu$. Loop filter $H(z)$ transforms its input signal into the control voltage to adjust the VCO and its transfer function is given by (5).

The operation involved in realizing $(11)$ is very simple, making the proposed algorithm desirable for VLSI implementation.

\subsection{Performance analysis}

\section{(1) Acquisition range}

It can be obtained from (8) that the first pair of zero-crossing points of $S\left(f_{d}\right)$ are located at $\pm 1 / T$. Besides, it is known from (10) that $\chi\left(f_{d}\right)>0$ for $\left|f_{d}\right|<1 / T$. Therefore, the acquisition range of the proposed method equals $2 / T$, regardless of the modulation order and the PN code length. This property makes the presented algorithm an attractive alternative for the carrier recovery of MDPSK DS/CDMA receiver undergoing sizable frequency offset.

\section{(2) Transient acquisition behavior}

To illustrate the transient acquisition behavior of the modified Scurve, computer simulations are carried out to obtain the trajectories of (5) and (11), using a 16DPSK DS/CDMA receiver with the symbol rate of $T=5 \mathrm{e} 5 \mathrm{symbol} / \mathrm{s}$ and the initial frequency offset of $f_{d, 0}=-0.99 / T$. Adopting the same $m$-sequence $(N=255)$ and the same step size $(\mu=0.01)$, the typical acquisition behaviors under $E_{s} / N_{0}=1 / 2 \sigma_{0}^{2}=-5$ and $10 d B$ are shown in Fig.4, which explicitly indicate that (11) has much shorter acquisition time [5] than (5). Both theoretical analysis and computer simulation demonstrate that with the same system parameters, the modified Scurve has superior acquisition performance to its counterpart.
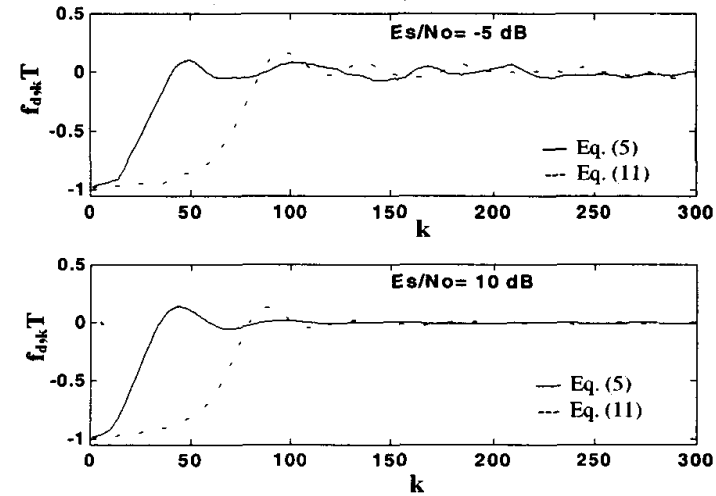

Figure 4. Transient acquisition behaviors resulted from different $S$-curves

\section{(3) Steady-state performance}

To analyze the influence of Gaussian noise on the steady-state performance of the proposed method, split $\nabla_{k}$ into the sum of $S\left(f_{d . k}\right)$ and the remaining noise term $\hat{w}_{k}$, and rewrite (11) as

$$
f_{d, k+1}=(1+\rho) f_{d, k}-\rho f_{d, k-1}+\mu \bar{S}\left(f_{d, k}\right)+\mu \chi\left(f_{d, k}\right) \hat{w}_{k}
$$

At the steady state when $f_{d . k}$ is sufficiently small, the linear approximation $\bar{S}\left(\hat{f}_{d, k}\right)=K_{A} \hat{f}_{d, k}$ holds true with

$$
K_{A}=\lim _{f_{f} \rightarrow 0} \frac{\partial \bar{S}\left(f_{d}\right)}{\partial f_{d}}=\frac{\pi T_{c} \chi_{0}}{6}\left(N^{2}-1\right)
$$

where $\chi_{0}=\left.\chi\left(f_{d}\right)\right|_{f_{d}=0}$. As a result, (12) can be approximated by

$$
f_{d . k+1}=\left(1+\rho+\mu K_{A}\right) \cdot f_{d, k}-\rho \cdot f_{d, k-1}+\mu \chi_{0} \cdot \hat{w}_{k}
$$

In (14), $\hat{w}_{k}$ and $f_{d . k}$ can be viewed as the input and output of a linear time-invariant system that is described by 


$$
Q\left(z^{-1}\right)=\frac{\mu \chi_{0} z^{-1}}{1-\left(1+\rho+\mu K_{A}\right) z^{-1}+\rho z^{-2}}
$$

Moreover, using the standard methods of [11], $\left\{\hat{w}_{k}\right\}$ can be proved to be a discrete, wide-sense stationary random process. Utilizing these conditions, we finally have

$$
E\left[f_{d}\right]=0
$$

and

$$
\sigma_{f_{d} T}^{2}=T^{2} \sigma_{\hat{u}}^{2} P_{Q}
$$

where $\quad \sigma_{\hat{k}}^{2}=\lim _{f_{d, c \rightarrow 0}} E\left[\hat{w}_{k}^{2}\right] \quad, \quad \sigma_{f_{d} T}^{2}=T^{2} \lim _{f_{d, k \Delta 0}} E\left[f_{d, k}^{2}\right] \quad$ and $P_{Q}=\frac{1}{2 \pi j} \oint_{C} Q(z) Q\left(z^{-1}\right) z^{-1} d z$. Eq.(16) indicates our AFC algorithm is unbiased and (17) gives its normalized variance of frequency jitter. We compare (17) with the CRB [12] as well as the performances of DAFF, DAFF and NDAFB [2] over the equivalent observation length $L_{E}$ [5]. Using the model of the equivalent estimator of [5], $L_{E}$ of the proposed algorithm can be computed through $L_{E}=\left(4 \pi \chi_{0} T c / K_{A}\right)^{2} / P_{Q}$. Without loss of generality, the comparison outcome for $L_{E}=50$ and 100 are shown in Fig.5, along with the simulation result of the proposed algorithm based on the modified S-curve plotted in Fig.2. The system parameters corresponding to $L_{E}=50$ are: $T c=2.56 \mathrm{e} 9 \mathrm{bps}, \rho=0.91, \mu=0.1$. The parameters corresponding to $L_{E}=100$ are: $T c=1.28 \mathrm{e} 9 \mathrm{bps}, \rho=0.82$, $\mu=0.19$. Examination of this figure indicates the simulation result coincides with the theoretical value quite well. Furthermore, throughout low to moderate level of SNR, the performance of the presented algorithm approaches the CRB more closely and it thus has more accuracy than those phase-based methods previously used for a fully-digital MDPSK DS/CDMA receiver.
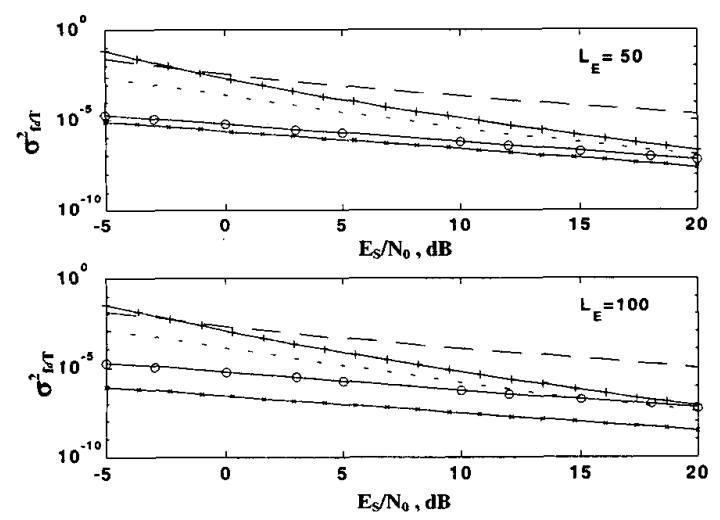

$$
\begin{aligned}
- & \text { NDAFB } \\
+- & \text { NDAFF } \\
\ldots . & \text { DAFF } \\
- & \text { Theoretical value of proposed algorithm } \\
0 & \text { Simulation result of proposed algorithm } \\
-x- & \text { CRB }
\end{aligned}
$$

Figure 5. Comparison of normalized variance of frequency jitter

\section{CONCLUSION}

In this paper, a non-phase-based AFC algorithm for a fully-digital MDPSK DS/CDMA receiver has been derived. This algorithm employs the improved gradient method and modified S-curve to correct the frequency offset within the range of twice the symbol rate. Digital implementation of the proposed method is also addressed and the simplicity of the algorithm makes it a desirable alternative for VLSI implementation. Both analytical evaluation and computer simulation have demonstrated that the proposed method outperforms previous phase-based methods not only in the acquisition range but also in the steady-state accuracy.

\section{REFERENCES}

[1] A. J. Viterbi, CDMA: principles of spread spectrum communication, Addison-Wesley Co., NY, 1995.

[2] O. C. Mauss, F. Classen and H. Meyr, "Carrier frequency recovery for a fulty digital direct-sequence spread-spectrum receiver: A comparison", Conf. Rec. VTC'93, pp. 392-395, 1993.

[3] F. D. Natali, "AFC tracking algorithms", IEEE Trans. Commun., Vol.32, No.8, pp. 935-947, Aug. 1984.

[4] D. Taich and I. Bar-David, "Maximum likelihood estimation of phase and frequency of MPSK signals", IEEE Trans. Inform. Theory, Vol.45, pp.2652-2654, Nov. 1999.

[5] U. Mengali and A. N. D'Andrea, Synchronization techniques for digital receivers, Plenum Press, 1997.

[6] A. N. D'Andrea and U. Mengali, "Design of quadicorrelators for automatic frequency control systems", IEEE Trans. Commun., vol.41, pp.988-997, Jun. 1993.

[7] M. L. Fowler and J. A. Johnson, "Extending the threshold and frequency range for phase-based frequency estimation", IEEE Trans. Signal Processing., vol.47, pp.2857-2863, Oct. 1999.

[8] A. Wannasarnmaytha, S. Hara and N. Morinaga, "Two-step Kalman-Filter-Based AFC for Direct Coversion-type receiver in LEO satellite Communications", IEEE Trans. Commun., vol.49, pp.246-253, Jan. 2000.

[9] J. G. Proakis, Digital communications, Third Edition, McGrawHill, Inc., 1995.

[10] V. Solo and X. Kong, Adaptive signal processing algorithms, Prentice Hall, Inc., Englewood Cliffs, NJ, 1995.

[11] A. V. Oppenheim and R. W. Schafer, Discrete-time signal processing, Prentice Hall, Inc., Englewood Cliffs, NJ, 1989.

[12] A. N. D'Andrea, U. Mengali and R. Reggiannini, "The modified Cramer-Rao bound and its application to synchronization problems", IEEE Trans. Commun., vol.42, pp.1391-1399, Feb./Mar./April 1994. 